University of Nebraska - Lincoln

DigitalCommons@University of Nebraska - Lincoln

P. F. (Paul Frazer) Williams Publications

Electrical \& Computer Engineering, Department

December 1990

\title{
Surface Flashover of Silicon
}

\author{
Frank E. Peterkin \\ University of Nebraska - Lincoln \\ Tim Ridolfi \\ University of Nebraska - Lincoln \\ Lonnie L. Buresh \\ University of Nebraska - Lincoln \\ Brian J. Hankla \\ University of Nebraska - Lincoln \\ D. K. Scott \\ University of Nebraska - Lincoln
}

See next page for additional authors

Follow this and additional works at: https://digitalcommons.unl.edu/elecengwilliams

Part of the Electrical and Computer Engineering Commons

Peterkin, Frank E.; Ridolfi, Tim; Buresh, Lonnie L.; Hankla, Brian J.; Scott, D. K.; Williams, P. F.; Nunnally, William C.; and Thomas, B. L., "Surface Flashover of Silicon" (1990). P. F. (Paul Frazer) Williams Publications. 12.

https://digitalcommons.unl.edu/elecengwilliams/12

This Article is brought to you for free and open access by the Electrical \& Computer Engineering, Department of at DigitalCommons@University of Nebraska - Lincoln. It has been accepted for inclusion in P. F. (Paul Frazer) Williams Publications by an authorized administrator of DigitalCommons@University of Nebraska - Lincoln. 


\section{Authors}

Frank E. Peterkin, Tim Ridolfi, Lonnie L. Buresh, Brian J. Hankla, D. K. Scott, P. F. Williams, William C. Nunnally, and B. L. Thomas 


\title{
Surface Flashover of Silicon
}

\author{
FRANK E. PETERKIN, TIM RIDOLFI, LONNIE L. BURESH, BRIAN J. HANKLA, D. K. SCOTT, \\ P. FRAZER WILLIAMS, MEMEBER, IEEE, WILLIAM C. NUNNALLY, MEMBER, IEEE, \\ AND B. L. THOMAS
}

\begin{abstract}
The development of high-voltage semiconductor devices has been hampered by the occurrence of flashover at the surface of the semiconductor. The physical mechanisms responsible for this phenomenon are not understood. We present new empirical information which clarifies the processes responsible for surface flashover in a vacuum ambient by showing clearly that in flashover current flows primarily inside the semiconductor surface rather than in the ambient. This observation is in fundamental disagreement with the standard model for vacuum flashover of insulator surfaces.
\end{abstract}

\section{INTRODUCTION}

$\mathrm{T}$ HERE HAS BEEN considerable interest recently in the application of semiconductors to high-voltage switching technology [1], [2]. A primary problem is that in most cases the switches flashover at the surface under average applied fields much less than the bulk breakdown field of the semiconductor. This problem has plagued the development of high-voltage solid-state devices for more than 30 years [3], but the physical basis for this flashover phenomenon is still not understood [2], [4]-[10].

In this paper we present new empirical information about surface flashover of silicon in a vacuum ambient which shows that in breakdown the current is carried primarily inside the semiconductor. This observation shows that the physical processes responsible for flashover of silicon are fundamentally different than those assumed in the standard model for flashover of insulators [11], [12]. When breakdown occurs we observe, as have other workers, visible emission from a plasma in the ambient just outside the silicon surface. This plasma may influence the course of the breakdown, but it does not appear to be the cause of the breakdown event. This evidence supports the earlier suggestion of Williams and Peterkin [6] that surface flashover might be caused by carrier accumulation at the semiconductor surface as a result of electric-field-induced band bending, and that of Thomas and Nunnally [10] that current filamentation in the semiconductor surface plays an important role in the process

Manuscript received January 31, 1990; revised May 29, 1990. This work was supported under Grant AFOSR-89-0253 from the U. S. Air Force Of fice of Scientific Research; F. E. Peterkin was partially supported by a U. S. National Science Foundation Scholarship, and B. L. Thomas was partially supported by the Strategic Defense Initiative Office of Innovative Science and Technology.

F. E. Peterkin, T. Ridolfi, L. L. Buresh, B. J. Hankla, D K. Scott and P. F. Williams are with the Department of Electrical Engineering, University of Nebraska-Lincoln, Lincoln, NE 68588-0511.

W. C. Nunnally and B. L. Thomas are with the Center for Energy Conversion Research, University of Texas at Arlington, Arlington, TX 76019. IEEE Log Number 9038833

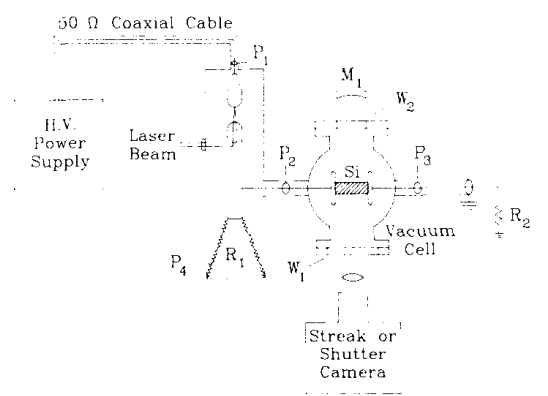

Fig. 1. Schematic diagram of the experimental setup

\section{Experimental Setup}

Fig. 1 shows the experimental setup we used. For all results reported here the sample was a rectangular prism of nominally intrinsic silicon with long dimension $10 \mathrm{~mm}$. The other two dimensions varied a little between samples and were about $7 \times 2 \mathrm{~mm}$. The samples were weakly n-type, with a resistivity as measured with a four-point probe of $1.3-1.6 \mathrm{k} \Omega \cdot \mathrm{cm}$, implying a carrier density of about $3 \times 10^{12} \mathrm{~cm}^{-3}$. All samples were ground flat, etched chemically with an HF-based ethcing solution, washed in deionized water, and blown dry with nitrogen. The etchant polished the sample chemically, leaving a shiny surface with considerable "orange peel." The sample under test was mounted between two parallel-plane copper electrodes, and contact was made by bonding an indium solder alloy (Indium Corp. Alloy \#1E) directly to the silicon using an ultrasonic soldering iron. Low-voltage $I-V$ curves of samples prepared this way were straight lines with slope corresponding to a sample resistivity of about $1.4 \mathrm{k} \Omega \cdot \mathrm{cm}$. The assembly was then indium soldered to a holder in the vacuum cell. The cell was evacuated with a small turbomolecular pump and was typically operated at a vacuum of about $10^{-7}$ torr.

Voltage was applied to the sample in pulses. The pulse generator consisted of a laser-triggered spark gap which discharged a length of $50-\Omega$ coaxial cable into a matched $50-\Omega$ resistor, labeled $R_{1}$ in Fig. 1. When fired without the sample in place, the generator produced rectangular pulses of magnitude up to about $35 \mathrm{kV}$ with pulse length determined by the length of the charged coaxial cable. The pulse rise time depended on voltage, and was 15-20 ns for a $30-\mathrm{kV}$ pulse. Jitter was typically $2-3 \mathrm{~ns}$. One electrode of the sample cell was connected to the gener- 
ator as shown, and the other was connected to a $50-\Omega$ termination, labeled $R_{2}$. For all data reported here, a $30-\mathrm{kV}$ voltage pulse was applied across the $10-\mathrm{mm}$ length of the sample, making the average applied field $30 \mathrm{kV} / \mathrm{cm}$. Capacitive divider voltage probes, labeled $P_{1}, P_{2}$, and $P_{3}$, monitored the voltage on the charged electrode of the laser-triggered spark gap, and on both sides of the sample. When used with a Tektronix 7834 oscilloscope, these probes had overall rise times of about 1,1 , and 2 ns, respectively. The total sample current could be determined from probe $P_{3}$ by dividing the output voltage by $50 \Omega$. The current in $R_{1}$ was monitored using $P_{4}$ to measure the voltage across a section of the distributed load.

Optical access to the front and back of the sample was provided by 4.5 - and 2 -in diameter fused quartz windows, labeled $W_{1}$ and $W_{2}$, respectively. The sample was mounted so that the broad faces of the sample were visible through the two windows. A locally constructed shutter camera and a Hammamatsu C979 streak camera were used to record the temporal and spatial development of optical events in the sample chamber. Both cameras were capable of near-single-photon detection sensitivity. The shutter camera had a minimum shutter time of about $5 \mathrm{~ns}$. The time resolution of the streak camera depended on the streak speed and the width of the entrance slit, and varied from less than $1 \mathrm{~ns}$ to about $15 \mathrm{~ns}$. The time scales of the electrical and optical diagnostics could be synchronized to within about \pm 1 ns, but in many cases the effective time synchronization was limited by the temporal resolution of the camera. In most experiments a spherical mirror, labeled $M_{1}$, was placed behind $W_{2}$ to provide an inverted image of the back side of the sample which could be recorded by the cameras simultaneously with the image from the front side. The effective optical aperture for all photos was determined by the focusing lens, and was $f / 8$.

\section{Experimental Results}

Fig. 2 shows a sequence of shutter photographs of the emission recorded during flashover, and a typical breakdown current oscillogram. The sample had undergone fewer than 30 shots. Except for changes in the delay between the arrival of the voltage pulse and the onset of the rapid current rise signaling breakdown, photos and current traces from samples which had undergone many more shots (up to 1000) were similar. In many cases the "aged" samples developed preferred breakdown paths whereas the breakdown path for "new" samples varied randomly from shot to shot. Both front and rear views are shown in Fig. 2 , with the rear view being inverted by the optical system. The positions of the edges of the sample for both views are indicated in the figure by white dotted lines. The shutter camera was capable of acquiring only one photo at a time, so these photographs are each of a different shot. There was substantial shot-to-shot variation, but the photos are representative of the sequence of events. The shutter time was $\sim 5 \mathrm{~ns}$, and the time when each photograph was taken is shown in the representative current trace in Fig. 2(d).

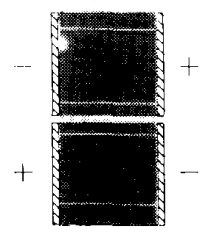

(a)

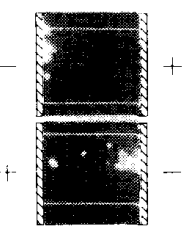

(b)

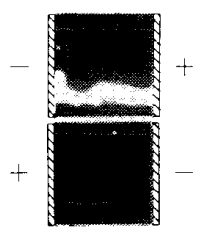

(c)

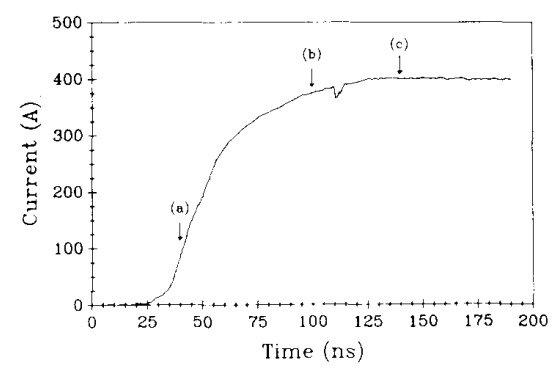

(d)

Fig. 2. Shutter photographs (a), (b), and (c) and a representative current trace (d) showing the progress of surface flashover in a vacuum ambient on a silicon sample which had undergone $<30$ shots. The applied voltage during the pulse was $30 \mathrm{kV}$ across a $1-\mathrm{cm}$ sample. Each photograph is from a different shot, and the current trace is that recorded for the photo in (c). The timing of each photo is shown by the vertical arrows in the current trace. A spherical mirror was used behind the sample to provide a simultaneous record of events on both the front and rear faces of the sample. The image from the rear face is inverted. In the photos, the upper photo is from the front face, the lower from the rear. In each photo, the boundaries of the sample are indicated by the white, dotted lines.

Fig. 3 shows streak photographs of two breakdown events. The horizontal dimension of the photos just spans the length of the sample, and time increases downward. In order to see events from the entire surface of the sample, a cylindrical lens was used to focus emission into a narrow, slit-shaped region. Since no entrance slit was used, the time resolution was determined from the dimensions of this region and was about $\pm 4 \%$ of full scale or 3.5 and 14 ns for Fig. 3(a) and (b), respectively. In Fig. 3(a) a spherical mirror was used behind the sample to record the emission from both broad faces. Since the image of the rear face is inverted, a front moving from left to right appears to move from right to left. This feature helps to separate events occurring on the two faces. In Fig. 3(b) emission is seen from only the front face because the rear mirror was blocked.

The first optical event observable in Figs. 2(a) and 3(a) is a small spot of light appearing near the cathode about $20 \mathrm{~ns}$ after the arrival of the voltage pulse at the sample. By reversing the polarity of the applied voltage pulse we verified that the spot is associated with the cathode and not with a specific contact on the sample. In all cases for which we have data, this first emission appeared after (typically $-10 \mathrm{~ns}$ ) the start of the rapid current rise signaling breakdown. Within 10-50 ns after the appearance of the cathode spot, emission spreads to other areas of the sample surface, moving roughly as a wavefront with a speed in the range $1-5 \times 10^{7} \mathrm{~cm} / \mathrm{s}$. As seen in Fig. 3(b), 


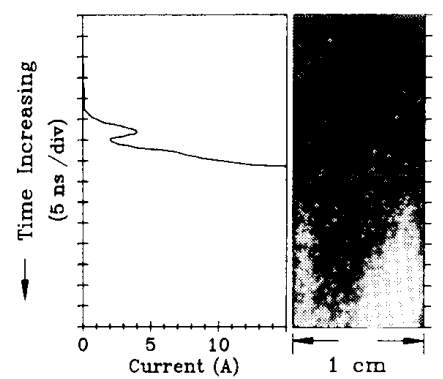

(a)

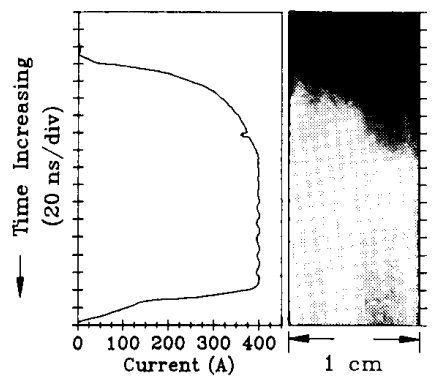

(b)

Fig. 3. Streak photographs and corresponding current traces of two breakdown events. The horizontal axis of the photos corresponds to the spatial dimension along the interelectrode axis, and the vertical to the temporal dimension, with time increasing downwards. The vertical edges of the photos correspond to the positions of the two contacts to the sample, and a cylindrical lens was used to concentrate emission to a thin line corresponding to $\pm 4 \%$, or about 0.7 division on each photo. The timing synchronization between photo and current trace is accurate to within this uncertainty. (a) Streak photo and current trace showing the early stage of breakdown. The white, dotted line is an artifact introduced to help in determining timing synchronization with the current trace. A spherical mirror was used behind the sample to provide a record of events on both faces of the sample simultaneously. Both images were focussed to a single line by the cylindrical lens. The image from the rear surface is in verted, making the front appear to travel in the opposite direction to that which it actually traveled. Fronts are seen on both faces in this photo, both emanating from the cathodic contact (on the left in the photo of the front surface). It is clear that visible emission first appeared some 10-15 ns after the onset of the rapid current rise leading to breakdown. (b) Lower temporal resolution streak photo and current trace showing the entire course of a breakdown event. In this case the rear mirror was blocked, and only emission from the front face is recorded. As in (a), the cathodic contact was on the left.

the motion was often sporadic, and the luminosity was nonuniformly distributed across the sample surface. Emission also often appeared in midgap, initially unconnected to either electrode. Fig. 2(b) shows a shutter photograph of such an event. Shutter photographs such as those shown in Fig. 2 show that the emission is localized to one, or at most a few, channels.

Fig. 4 shows typical oscillograms of the current in a sample during the earliest stage of breakdown, as measured by $P_{3}$. The trace in Fig. 4( $\left.a\right)$ was obtained from a "new" sample ( $<10$ shots $)$, and that in Fig. $4(b)$ from an "aged" sample which had been subjected to $\sim 1200$ shots. The first prominent peak in both traces is due to

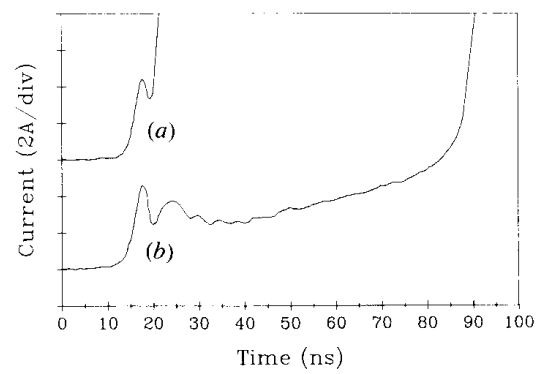

Fig. 4. Current traces of breakdown events in $(a)$ a "new" sample which had undergone $<10$ shots, and $(b)$ an "aged" sample which had undergone $\sim 1200$ shots. The initial peak is due to capacitive coupling and reflects the arrival of the voltage pulse at the sample.

capacitive coupling, and provides a convenient time marker since it results from the arrival of the voltage pulse at the sample. For the "new" sample, a rapid current increase signaling breakdown occurred within $10 \mathrm{~ns}$ of the arrival of the voltage pulse at the sample. For the "aged" sample, on the other hand, a current rising slowly from about 2.7 to more than $6 \mathrm{~A}$ flowed for $\sim 60 \mathrm{~ns}$ before the rapid current rise signaling breakdown appeared. For "aged" samples the delay time to breakdown varied randomly from shot to shot over a typical range of $100 \mathrm{~ns}$ or more. It is interesting that for a series of consecutive shots the portions of the current traces before the rapid current rise signaling breakdown were quite repeatable. On one such sample, these portions could not be distinguished from each other for traces from ten consecutive shots.

The initial sample current of $2.7 \mathrm{~A}$ seen in Fig. $4(b)$ is that expected from a sample with a volume resistivity of $2.3 \mathrm{k} \Omega \cdot \mathrm{cm}$. Low-voltage $I-V$ curves of the same sample, both when "new" and "aged" were straight lines with slope corresponding to a resistivity of $1.4 \mathrm{k} \Omega \cdot \mathrm{cm}$. This discrepancy may be due to the contacts, or it may reflect the thermal carrier generation rate. Current limitation due to the contacts would be consistent with our observation of luminous emission from the cathodic contact, and with the results of Donaldson et al. who found the electric field in a silicon sample under similar conditions to be nonuniform along the gap axis, with the largest field near the contacts.

The increase in breakdown delay with number of breakdown events evident in Fig. 4 was seen in all samples we studied. For one sample, after about 1000 shots breakdown almost never occurred during the 250-ns charging pulse. The "aging" effect could be partially reversed. The delay could be reduced substantially by simply leaving the sample under vacuum $\left(\sim 10^{-7}\right.$ torr $)$ overnight, or by admitting dry grade $\mathrm{N}_{2}, \mathrm{O}_{2}$, or atmospheric air to the cell. For these treatments, the sample could usually be "aged" again through the application of several hundred additional shots. The sample could sometimes be cycled in this way several times.

The effect on breakdown of exposure to air is shown in Fig. 5, where the delay times to breakdown are plotted for 50 consecutive shots for an "aged" sample which had 


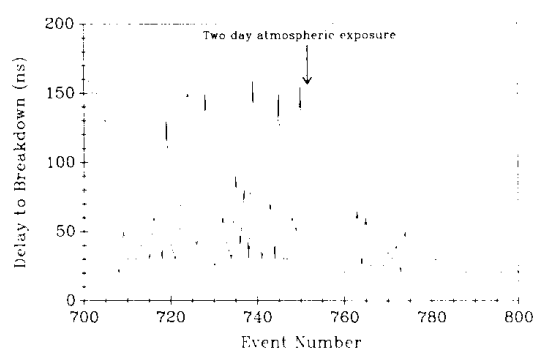

Fig. 5. Plot showing the delay between the application of the voltage pulse and the onset of the rapid current rise signaling breakdown for $100 \mathrm{con}$ secutive shots from a sample which had already undergone about 700 shots. At the point indicated by the vertical arrow experimentation was suspended, and room air was admitted to the cell. After two days the cell was then evacuated again, and the delay for 50 more shots recorded

already undergone about 700 shots. The delay for these shots is highly variable. Also shown in the figure are the delays for the following 50 shots obtained after the sample had been exposed to atmospheric air for two days. The delay is much less variable, and is clearly less on the average than before exposure to the air.

We observed an interesting effect of illumination on the breakdown of "aged" samples. In one such sample, breakdown almost never occurred in the absence of illumination during the $\sim 250$-ns voltage pulse. We carried out experiments in which a small portion of the laser beam used to trigger the laser-triggered spark gap in the main pulse generator was used to illuminate diffusely the front surface of the sample. A portion of the main beam was split off, attenuated, and directed into a diffuser. The light scattered from the diffuser uniformly illuminated the front surface of the sample located inside the test cell. The laser pulse was about $15 \mathrm{~ns}$ long and struck the sample 10-20 ns before the arrival of the high-voltage pulse. For intercepted illumination energy greater than some value which we estimate to be about $1 \mu \mathrm{J}$, breakdown occurred within $5 \mathrm{~ns}$ of the arrival of the high-voltage pulse at the sample. By reducing the energy below this value, the breakdown delay could be increased continuously. For a fixed laser illumination energy, the jitter in the breakdown delay was less than $5 \mathrm{~ns}$. Surprisingly, it appeared that the 1064-nm fundamental wavelength of the $\mathrm{Nd}$ : YAG laser was more efficient at inducing breakdown than was the $532-\mathrm{nm}$ second harmonic.

CW illumination from an incandescent 40-W light bulb in a desk lamp could also be used to decrease the breakdown delay time in an "aged" sample. For the sample shown in Fig. 5 after it had been "aged" and "rejuvenated" several times the delay was constant at $50+5 \mathrm{~ns}$. For full rated voltage on the lamp, breakdown occurred within $10 \mathrm{~ns}$ of the arrival of the high voltage pulse at the sample. The delay could be continuously increased by decreasing the lamp voltage.

\section{Discussion}

In the commonly accepted model, surface flashover of insulators in a vacuum occurs as a result of breakdown of gas desorbed from the surface of the insulator [11], [12].
Electrons emitted from the cathode are responsible for desorbing this gas and for ionizing it through electron impact excitation, and the flashover current flows in the resulting plasma. Our results show very clearly that this model does not describe flashover of our silicon samples. Besides ionizing the atoms of the desorbed gas, electron impact excitation also produces light emission, and such emission is observed coincidentally with significant current flow in vacuum flashover of insulators [13]. Microdischarges, a related phenomenon, are observed in prebreakdown activity of vacuum gaps [14]. These discharges are thought to result from gas ejected from the electrodes. Visible emission is observed from these discharges for currents of the order of $10 \mu \mathrm{A}$.

In all cases for which we have data, breakdown started and a current of several tens of amperes flowed before the appearance of any visible emission at all. Further, the sample current typically rose to a value $>300 \mathrm{~A}$ before emission appeared outside the region of the cathode spot, and remained between $300-400 \mathrm{~A}$ for $30-50 \mathrm{~ns}$ before the gap between the electrodes was bridged with luminescence. Such currents could not flow through a gas discharge as assumed in the standard model without inducing visible emission. Thus, the standard surface flashover model cannot describe flashover of our silicon samples.

Where is the current flowing? It is unlikely that currents of this magnitude could be carried by a beam of ballistic electrons in the vacuum. Transition radiation is generally observed at the anode of vacuum gaps for prebreakdown currents greater than about $1 \mathrm{~mA} / \mathrm{cm}^{2}$ [15]. We do not see such an emission from the anode of our gap, even though the current is many orders of magnitude larger than the reported threshold for emission of transition radiation. We conclude, therefore, that most of the current must flow in the semiconductor.

The mechanisms responsible for this current flow are not as clearly indicated. Williams and Peterkin have proposed the following model of surface breakdown in silicon [6]. Initially, there is a thin, conductive layer at the surface of the silicon sample. This layer is similar to the inversion layer in MOSFET transistors, and is produced by carrier accumulation at the surface as the result of band bending induced by a normal electric field [16]. The source of this field might be charge in an insulating surface layer (either fixed charge associated with surface states or impurities in a native oxide layer), or charge bound to the surface of the layer (perhaps through the action of electron impact). When voltage is applied to the sample, a thermal runaway process ensues in which ohmic heating of the conductive layer increases conductivity through increased thermal carrier generation. The ohmic heating increases in turn, leading to thermal runaway and breakdown. Although there are some difficulties, the results we report here are generally consistent with this model.

The question of the origin of the optical emission seen in the photographs presented here naturally arises. The most likely source is luminescence from gas evaporated from the semiconductor surface as the result of heating. 
Emission would result from electrical breakdown of the low-pressure gas or, possibly, from excitation associated with the evaporation event. A preliminary examination of the spectrum of the optical emission showed that it consists of discrete lines. The only line we were able to clearly identify was the $\mathrm{H} \alpha$ line of atomic hydrogen. Each shot caused a pressure rise in the cell corresponding to a liberation of $10^{14}-10^{15}$ atoms or molecules. Analysis of the gas with a residual gas analyzer showed mass components corresponding to $\mathrm{H}, \mathrm{H}_{2}, \mathrm{C}, \mathrm{O}, \mathrm{OH}, \mathrm{H}_{2} \mathrm{O}, \mathrm{N}_{2}$ or $\mathrm{Si}$, and $\mathrm{SiO}$. There were also a number of components which we believe to correspond to hydrocarbon compounds.

A simple calculation [6] shows that a uniform (nonfilamentary) heating process is unlikely to induce breakdown through thermal runaway at the surface on a time scale consistent with our results for "new" samples. For example, if heat flow out of the surface conduction layer is neglected, and a surface carrier density of $2 \times 10^{17}$ $\mathrm{cm}^{-3}$ assumed, then for an applied field of $30 \mathrm{kV} / \mathrm{cm}$ and other parameters the same as the room temperature bulk values for silicon, ohmic heating would result in a rate of temperature rise of about $2.5 \times 10^{10 \circ} \mathrm{C} / \mathrm{s}$. If we arbitrarily assume that breakdown occurs when the surface temperature has increased by $500^{\circ} \mathrm{C}$, we would predict a breakdown time of about $20 \mathrm{~ns}$. While this figure might be considered consistent with the 5-10 ns delay we observe from "new" samples, it is about the shortest that can be produced using reasonable values of the parameters involved, and more realistic estimates of the effect of heat conduction out of the surface layer and of reduced carrier mobility due to the surface, elevated temperature, and high carrier densities would certainly increase it.

The localized nature of the observed optical emission implies that the gas is evaporated from channels on the silicon surface, and suggests that current filamentation occurs inside the semiconductor. The assumption of filamentary current conduction in the silicon is consistent with surface damage patterns seen after a number of breakdown events, and is strongly supported by electron photomicrographs to be presented elsewhere of samples after flashover. Thomas and Nunnally have developed a model of breakdown in which it is assumed that current flows in thin filaments at the surface of the silicon [10], and they show that simple resistance heating in the filaments can account for breakdown on the time scale we observe.

On the basis of these considerations, we propose that the following sequence of events is responsible for inducing breakdown at the surface of our samples. First, a layer of enhanced conductivity exists at the surface of the silicon, as discussed by Williams and Peterkin [6]. When voltage is applied to the sample, current flows in this layer, resulting in roughly uniform heating. After some time, current constriction begins near the cathode, and current in this region becomes localized to one or more thin filaments. The enhanced conductivity at the tip of each filament induces increased heating there, and causes the filament to grow in length. Breakdown is complete when one or more filaments reach the opposite electrode.
The current trace from the "aged" sample shown in Fig. 4(b) is consistent with this model. The slowly increasing current observed during the first $60 \mathrm{~ns}$ of the voltage pulse is due partly to bulk conduction, and partly to conduction in the assumed surface layer. The rate of rise of current is much too rapid to be due to heating of the bulk material, and is probably due to heating in the surface layer. The observation that the current traces from consecutive shots were quite reproducible during this time is consistent with this uniform heating interpretation. About 65-70 ns after the start of the voltage pulse current we believe that constriction began near the cathode, and one or more current filaments started growing towards the anode. At this time, the current began to increase much more rapidly, and about $10 \mathrm{~ns}$ later visible emission appeared from the cathode region.

Current traces such as that in Fig. 2(d) are also consistent with our model. After an initial fast rise, the current rise slows and then becomes noticeably flat at a value corresponding to negligible sample resistance and complete breakdown. The delay time from the application of the voltage pulse until complete breakdown could usually be determined from such current traces to within 5-10 ns. In all cases for which we have data, this time of complete breakdown coincided to within about $10 \mathrm{~ns}$ with the time when the visible emission first continuously bridged the gap. Assuming the visible filament to be an indicator of the position of the underlying current filament(s), this observation supports the important role in breakdown played by current filamentation, as postulated by Thomas and Nunnally [10].

The physical mechanisms responsible for the optical triggering of breakdown which we observe remain unclear. Enloe and Gilgenbach have reported the optical induction of breakdown of a stressed insulator [17], but it is clear, for two reasons, that this is a different effect than that which we observe. First, we observe induction of flashover by 1064-nm laser radiation with a threshold fluence of less than $1 \mu \mathrm{J} / \mathrm{cm}^{2}$, and even by the radiation from a $40-\mathrm{W}$ incandescent light bulb; whereas Enloe and Gilgenbach report a threshold fluence of about $10 \mathrm{~mJ} / \mathrm{cm}^{2}$ of $248-\mathrm{nm}$ radiation. They show that the effect they observe is the result of multiphoton ionization of and subsequent electron emission from the surface of the insulator. It is very difficult to explain the much lower threshold fluence we observe with near-infrared light in terms of such a mechanism. Second, for the effect reported by Enloe and Gilgenbach flashover results from the charging of the insulator surface. Even if some charging process were operative in our case, the accumulated surface charge would be minimal because the dielectric relaxation time of our silicon samples is of the order of $1 \mathrm{~ns}$.

The results we report suggest that the triggering of breakdown is the result of enhanced conductivity near the surface of the sample, which would be consistent with our proposed model. The observation that near-infrared is more efficient than visible light in inducing the effect is surprising, however. Perhaps in the "aged" samples the surface is damaged sufficiently that light absorbed in the 
top layer does not contribute substantially to the conductivity. The infrared light would penetrate more deeply to undamaged material and result in a greater increase in current density.

The physical reasons for the "aging" effect seen in Fig. 4 also remain unclear. The most likely explanation of the effect is that a surface layer, probably an oxide, plays an important role in the breakdown. Charging of this layer could result in the surface-normal fields postulated by Williams and Peterkin [6]. Repeated flashover events might remove this layer and damage the silicon surface, thereby reducing the conductivity of this surface layer and delaying the onset of breakdown. The procedures for reversing "aging" would tend to regrow this oxide layer on the damaged surface. An alternate explanation is based on surface damage. Each breakdown event damages some portion of the surface of the silicon. Damage would reduce carrier mobility in the inversion layer and could introduce carrier traps. Both effects would reduce the surface heating rate, thereby increasing the delay to breakdown. This latter explanation is consistent with the difference we observe between the effects of visible and infrared illumination on breakdown.

\section{Summary}

Our results clearly show that the standard model of surface flashover of insulators in vacuum [11], [12] does not describe surface flashover of silicon in a vacuum ambient. The physical mechanism(s) responsible for flashover of silicon are not indicated as clearly, but it appears that breakdown proceeds through heating of the silicon surface, followed by current constriction in this layer, and the growth of current filaments. These results support and extend the general models proposed by Williams and Peterkin [6] and by Thomas and Nunnally [10].

\section{ACKNOWLEDGMENT}

The authors have had a number of useful conversations with B. J. Ganguly and A. Garscadden regarding preparation of silicon samples, vacuum systems, and analysis of optical emission spectra. They wish to thank A. J. Gibbs for helpful advice on chemical etching of silicon, and N. J. Ianno and G. G. Myer for advice on several aspects of silicon processing techniques. They also wish to thank R. A. Dougal and M. A. Gundersen for helpful discussions regarding high-current, vacuum, and lowpressure discharges, respectively.

\section{REFERENCES}

[1] See for example W. C. Nunnally, "Photoconductive power switches, a review." in Dig. Tech. Papers, 5th Pulsed Power Conf., M. F. Rose and P. J. Turchi, Eds. New York, NY: IEEE, 1985, pp. 235241 .

[2] G. M. Loubriel, M. W. O'Malley, and F. J. Zutavern, "Toward pulsed power uses for photoconductive semiconductor switches: Closing switches," in Dig. Tech. Papers, 6th IEEE Pulsed Power Conf., P. J. Turchi and B. H. Bernstein, Eds. New York, NY: IEEE, 1987, pp. 145-148.

[3] C. G. B. Garrett and W. H. Brattain, "Some experiments on, and a theory of surface breakdown," J. Appl. Phys., vol. 27, p. 299, 1956.

[4] - "Physical theory of semiconductor surfaces," Phys. Rev.. vol. 99, p. $376,1955$.
15] B. L. Thomas and W. C. Nunnally, "Investigation of surface flashover in silicon photoconductive power switches, " in Dig. Tech. Papers, 6th Pulsed Power Conf., P. J. Turchi and B. H. Bernstein, Eds. New York, NY: IEEE, 1987, pp. 149-152.

[6] P. F. Williams and F. E. Peterkin, "A mechanism for surface flashover of semiconductors," in Dig. Tech. Papers, 7th Pulsed Power Conf., B. H. Bernstein and J. P. Shannon, Eds. New York, NY: IEEE, 1989, pp. 890-892.

[7] B. L. Thomas and W. C. Nunnally, "Recent developments in the investigation of surface flashover on silicon photoconductive power switches." in Dig. Tech. Papers, 7th Pulsed Power Conf., B. H. Berstein and J. P. Shannon, Eds. New York, NY, IEEE 1989, pp. $893-896$.

[8] S. H. Nam and T. S. Sudarshan, "Observation of three distinct phases leading to pulsed surface flashover along silicon in vacuum, " in Proc. XIIIth Int. Symp. on Discharges and Electrical Insulation in Vacuum (Paris, France, 1988), pp. 527-530.

[9] _- "Effect of teakage current and light emission on surface flashover along silicon in vacuum," in Dig. Tech. Papers, 7th Pulsed Power Conf., B. H. Bernstein and J. P. Shannon, Eds. New York, NY: IEEE, pp. 362-364

[10] B. L. Thomas, "Surface flashover in silicon photoconductive power switches," Ph.D. dissertation, University of Texas at Arlington, Arlington, TX, 1989.

[11] R. A. Anderson and J. P. Brainard, "Mechanism of pulsed surface flashover involving electron-stimulated desorption." J. Appl. Phys. . vol. 51, p. $1414,1980$.

[12] R. A. Latham, High Voltage Vacuum Insulation: The Physical Basis. London, UK: Academic Press, 1981, pp. 229-240.

[13] J. D. Cross, "High speed photography of surface flashover in vacuum," IEEE Trans. Electron. Insulation, vol. EI-13, p. 145, 1978.

[14] P. A. Chatterton, "Vacuum breakdown," in Electrical Breakdown of Gases, J. M. Meek and J. D. Craggs, Eds. New York, NY: Wiley, 1978.

[15] C. J. Bennette, L. W. Swanson, and R. W. Strayer, "Visible radiation from metal anodes preceding electrical breakdown," J. Appl. Phys. vol. 35, p. 3054, 1964.

[16] See for example E. H. Nicollilan and J. R. Brews, MOS Physics and Technology. New York, NY: Wiley, 1982.

[17] C. L. Enloe and R. M. Gilgenbach, "Microscopic and macroscopic material property effects on ultraviolet-laser-induced flashover of an gled insulators in vacuum," IEEE Trans. Plasma Sci., vol. 16, p. $379,1988$.

Frank E. Peterkin was born in Omaha, NE, in 1964. He received the B.S and M.S. degrees in electrical engineering from the University of $\mathrm{Ne}$ braska-Lincoln in 1986 and 1988, respectively. As a National Science Foundation Fellow, he studied the physical mechanisms initiating breakdown in high voltage trigatron spark gaps for his master's thesis research.

Currently he is working towards the Ph.D. degree in electrical engineering at the University of Nebraska. His research is centered on understanding the surface breakdown of semiconductors used in high-power photoconductive switching.

Tim Ridolfi was born in Lincoln. NE, on November 5, 1965. He has pursued electrical engineering at the University of Nebraska-Lincoln since 1984; he recived the B.S. degree in 1988 and is a candidate for the M.S. degree in 1990.

His current research interest is the imaging of electric fields by means of polarization modulation due to the Kerr effect.

Lonnie L. Buresh was born in Lincoln, NE, on October 16, 1968. He is currently working towards the B.S. degree in electrical engineering at the University of Nebraska-Lincoln.

His major area of study is systems and controls. 
Brian J. Hankla is a senior in electrical engineering at the University of Nebraska-Lincoln. He earned a technical degree in electronics prior to university enrollment and worked in industrial thick-film processing.

D. K. Scott, photograph and biography not available at the time of publication.

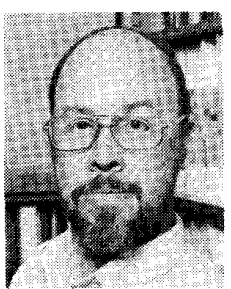

P. Frazer Williams (M'78) received the B.S. degree in physics from the California Institute of Technology, Pasadena, in 1967, and the Ph.D. degree in electrical engineering from the University of Southern California, Los Angeles, in 1973.

His professional experience includes: M.T.S. Bell Laboratories, Murray Hill, NJ; Assistant Professor in the Department of Physics, University of Puerto Rico, Rio Piedras, PR; and Assistant and Associate Professor of Electrical Engineering, and Associate Professor of Physics,

Texas Tech University, Lubbock, TX. He is currently the Lott College Professor of Electrical Engineering at the University of Nebraska-Lincoln. His current research interests center around surface flashover of semiconductors, and the electrical breakdown of gases. Past interests include resonant and nonresonant Raman scattering from semiconductors and simple molecular gases, and the dielectric response of quasi-one-dimensional conductors. He has published about 50 technical papers.

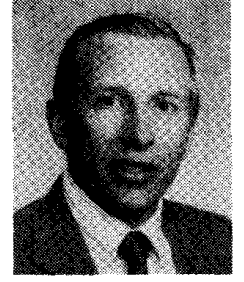

William C. Nunnally (S'67-M'69) was born in El Paso, TX, on March 5, 1946. He received the B.S.E.E. degree from Texas Tech University, Lubbock, in 1969. He received the M.S.E.E. degree in 1971 and the Ph.D. degree in 1975 from Texas Tech University.

He joined the staff of the Los Alamos National Laboratory in 1974 as an Engineer in the magnetic fusion research group. During his tenure at Los Alamos, his areas of work included laser fusion, laser isotope separation, high speed-optical-semiconductor electronics, particle accelerator power systems, magnetic switching, and photoconductor switching. He served as the Director of the Center for Energy Conversion Research at the University of Texas at $\mathrm{Ar}$ lington from June 1985 until September 1987, when he joined the Faculty of the University of Texas at Arlington as an Associate Professor, and continued as Director until February 1988. His research interests include applications of lasers and semiconductors, high-speed power optoelectronics, electromagnetic launchers, optical fiber applications, and laser diagnostics.

B. L. Thomas, photograph and biography not available at the time of publication. 